\title{
Polymer-polymer interaction parameters by inverse gas chromatography: a novel molecular interpretation of non- random partitioning of solvent probes in polymer blends
}

\author{
Zhanjie Tan, G. Julius Vancso* \\ Faculty of Chemical Technology, University of Twente, P.O. Box 217, \\ 7500-AE Enschede, The Netherlands
}

(Received: March 28, 1996; revised manuscript of August 27, 1996)

\begin{abstract}
SUMMARY:
Non-random partitioning of molecular probes in a polymeric mixture was examined with Kirkwood-Buff-Zimm (KBZ) cluster integrals. Equations were derived to give relationships of thermodynamic quantities that can be obtained by using inverse gas chromatography (IGC). The mer-mer correlation structure factor $S^{*(A B)}(0)$ in a binary mixture of polymers $A$ and $B$ in terms of the spatial distribution of a probe, $S$, around a molecule $A$ and a molecule $\mathrm{B}$ is discussed. The derivation allows a direct assessment of the correlation structure factor obtained either from scattering experiments or from IGC measurements. The common polymer-polymer interaction parameter from the two types of experiments is discussed with respect to criticism concerning IGC. The detailed molecular description of the probe behavior in a polymeric mixture at infinite dilution of the probe given in this work can be used in IGC studies of the microstructure of polymer mixtures or other mixtures of liquids. In order to illustrate our approach, the structure factor and the interaction parameter of the blends of polystyrene (A) and poly(butyl methacrylate) (B) were evaluated using IGC results of DiPaola-Baranyi and Degre.
\end{abstract}

\section{Introduction}

The non-random partitioning of molecular probes in polymer-solvent systems has been a major problem in studies of thermodynamics and structure by molecular probe techniques $^{1,2)}$. One of the most common problems concerns the probe dependence of the value of the polymer-polymer interaction parameter as determined by inverse gas chromatography (IGC), one of the molecular probe techniques. Attempts have been made to tackle this problem ${ }^{3-5)}$. However, a direct relationship between the probe non-random partitioning and its effect on the polymer-polymer interaction parameter has not been established. Such a relationship would not only help us to interpret results of IGC measurements on blends and understand the microstructure and interaction parameters in a mixture, it would also bring together the fundamentally different ways of determining thermodynamic parameters on a molecular level, i.e. scattering experiments and thermodynamic experiments with molecular probes $^{6)}$.

In this article we apply Kirkwood-Buff cluster integrals ${ }^{7)}$ in order to characterize the non-random partitioning of probes in a mixture. Employing the Kirkwood-BuffZimm (KBZ) cluster integral method by Ben-Naim ${ }^{8)}$, Newman ${ }^{9)}$ and Horta ${ }^{10)}$, we derived thermodynamic relationships between the preferential solvation of probes at their infinite dilution in a binary mixture and the correlation structure factor of the 
mixture, which can be directly determined from either scattering experiments ${ }^{11-13)}$ or by IGC experiments. We give an interpretation of non-random partitioning with respect to local compositions. In addition, we discuss the fundamental assumptions dealing with IGC data interpretation for polymer blends.

We conclude that the approach presented in this work provides a good way of investigating the non-random partitioning and treating experimental data obtained by IGC. Our goal is to establish a way of determining the structure factor of polymeric mixtures, and hence the interaction parameters.

In this paper the non-random partitioning is defined as follows. A molecule, when it is dissolved in a polymer blend, does not probe the average composition in the mixture. It "feels" a local composition of the different components. The local composition depends on the fluctuation of both the particle density and the chemical composition of the blend, as well as on the interactions of the probe with the different components.

\section{Cluster integrals and non-random partitioning characterized by preferential solvation}

The Kirkwood-Buff cluster integral is defined as the integral of the pair correlation function minus unity over a system,

$$
G_{i j}(0)=\int_{V}\left[g_{i j}(R)-1\right] \mathrm{d} \boldsymbol{R}
$$

which forms a $\boldsymbol{G}$ matrix for a multi-component system ${ }^{14,15)}$. In this definition, $\boldsymbol{R}$ stands for the vector that describes the relative position of the mass centers of molecules 1 and 2 in the system (i.e., $R=R(2)-\boldsymbol{R}(1)$ ). The physical significance of a Kirkwood-Buff cluster integral is related to the overall excess (or deficiency) of a given type ( $i$ ) around a single "typical" molecule $j$ compared to the average. In order to understand the direct (short-range) and the indirect (long-range) correlation, one may use the total correlation function as defined by,

$$
h_{i j}(\boldsymbol{R})=g_{i j}(\boldsymbol{R})-1
$$

in Eq. (1) as suggested by Ornstein and Zernike ${ }^{16)}$. The term $\frac{G_{i j}(0)-G_{i j}(0)}{\bar{V}_{i j}}$ corresponds to the specific preferential solvation of component $j$ by a molecule of species $i$ over species $l$ when a $j$ molecule is dissolved in the bulk of a multi-component mixture ${ }^{8,17)}$. Indirectly, it characterizes the compatibility of the inserted molecule with one component over another component in the mixture in terms of spatial distribution. The value of the preferential solvation is useful for discussing the specific interactions of a species with the other components in a mixture, and is often used in discussions of solvation in ionic or polar mixtures of small molecules ${ }^{9,18}$ ). It is a very important quantity for describing the non-random partitioning of probe molecules in a mixture, as will be shown below. 


\section{Mer-mer correlation structure factor and preferential molecular solvation}

\section{Molecular correlation and preferential molecular solvation}

When the probe concentration in an IGC experiment approaches zero, a ternary system returns to a binary system. A thermodynamic formula under this condition will contain separated thermodynamic quantities of the binary system, which can be determined by using a molecular probe technique. For the cluster integrals at infinite dilution of one of the components in a multi-component system, Ben-Naim derived the necessary equation. This formula can be used, for example, in dealing with the relationship of the cluster integrals and the measurable thermodynamic functions ${ }^{19}$ ). It is interesting to mention that the same expression was derived and used in studies of free energy of transfer of a component from one state to another in electrolytic solutions ${ }^{9)}$ and supercritical fluids ${ }^{18,20)}$.

Eq. (3) can be derived at infinite dilution of one of the components ${ }^{8,9,21,22)}$ for a ternary system containing the solvent probe $\mathrm{S}$, polymer $\mathrm{A}$ and polymer $\mathrm{B}$,

$$
\frac{x_{A} x_{B}}{\bar{V} S^{A B}(0)}\left[G_{S B}^{\infty}(0)-G_{S A}^{\infty}(0)\right]=\lim _{\rho_{S} \rightarrow 0}\left[\frac{\partial \ln \left[{ }^{(w)} \gamma_{S}^{(M)} /^{(w)} \gamma_{S}^{(A)}\right]}{\partial x_{A}}\right]=\frac{1}{k T} \lim _{\rho_{S} \rightarrow 0}\left[\frac{\partial \Delta \mu_{S}}{\partial x_{A}}\right] \text { (3) }
$$

where ${ }^{(w)} \gamma_{S}^{(A)}$ and ${ }^{(w)} \gamma_{S}^{(M)}$ denote the probe activity coefficients (weight fraction based) in pure $\mathrm{A}$, and in the mixture of $\mathrm{A}$ and $\mathrm{B}$, respectively; $k$ is the Boltzmann constant, $\rho_{S}$ is the number density of the solvent probe molecules, $\mathrm{S}$, and the superscript $\infty$ stands for the infinite dilution of $S ; x_{A}$ and $x_{B}$ are the mole fractions of $A$ and $B$ polymers in the mixture, respectively; the value $\bar{V}\left(=x_{A} \bar{V}_{A}+x_{B} \bar{V}_{B}\right)$ is the molar volume of the polymer blend; $\Delta \mu_{S}$ stands for the free energy of transfer of the probe from the pure $\mathrm{A}$ to the $\mathrm{AB}$ mixture, and $S^{(A B)}(0)$, the molecular correlation structure factor of the polymer blend, is defined by ${ }^{11,23)}$,

$$
\left.S^{(A B)}(0)=x_{A} x_{B}\left[\frac{1}{R T} \frac{\partial \mu_{A}}{\partial \ln x_{A}}\right]^{-1}=N_{A}\langle\Delta x)^{2}\right\rangle
$$

for one mole of molecules of the mixture, where $N_{A}$ stands for the Avogadro number. The second equivalence is obtained when higher order fluctuation terms are neglected ${ }^{24)}$. A characteristic relationship of the cluster integrals is given by BenNaim's analysis ${ }^{21)}$,

$$
\phi_{A} G_{S A}^{\infty}(0)+\phi_{B} G_{S B}^{\infty}(0)=k T \kappa_{T}^{(A B)}-\bar{V}_{S}^{\infty}
$$

where $\kappa_{T}^{(A B)}$ is the isothermal compressibility of the blend and $\bar{V}_{S}^{\infty}$ is the partial molar volume of the probe. In Eq. (5), $\phi_{A}$ and $\phi_{B}$ stand for the volume fractions of the components $A$ and $B$, respectively. Experiments are usually conducted separately on the pure compounds and their mixtures in IGC at infinite dilution of the probes. Eq. (3) shows the direct relationship between the preferential solvation and the free energy of transfer of probes, such as solvents. In studies of polymers, volume changes due to fluctuations are usually important because they strongly affect the 
properties of polymeric mixtures ${ }^{25)}$. In the following section, we derive the relationship for the preferential solvation in terms of the volume fraction.

\section{Mer-mer correlation structure factor and the preferential molecular solvation}

At zero scattering angle, the reciprocal of the static structure factor is equal to the second concentration derivative of the free energy density ${ }^{3 a)}$,

$$
\left[S^{*(A B)}(0)\right]^{-1}=\frac{\partial^{2}(\Delta f /(k T))}{\partial \phi_{A}^{2}}
$$

$\Delta f$ is the free energy of mixing for a unit volume of mixtures. In terms of chemical potential $\Delta \mu_{A}$, the structure factor has the following form,

$$
S^{*(A B)}(0)=\phi_{A} \phi_{B}\left[\frac{1}{R T} \frac{\partial\left(\Delta \mu_{A} / \bar{V}_{A}\right)}{\partial \ln \phi_{A}}\right]^{-1}
$$

This quantity is known as the mer-mer correlation structure factor for the unit volume of the mixture. It describes the mer agglomeration, i. e.,

$$
S^{*(A B)}(0)=\left\langle(\Delta \phi)^{2}\right\rangle
$$

when the higher order fluctuation terms are neglected ${ }^{24)}$. It is related to the parameter $\chi_{S C}$ by the following equations,

$$
\begin{aligned}
& {\left[S^{*(A B)}(0)\right]^{-1}=\left[\frac{1}{\bar{V}_{A} \phi_{A}}+\frac{1}{\bar{V}_{B} \phi_{B}}-2 \chi_{S C}\right]} \\
& \chi_{S C}=-\frac{1}{2} \frac{\partial^{2}\left(\Delta f_{e x} /(k T)\right)}{\partial \phi_{B}^{2}}
\end{aligned}
$$

where $\Delta f_{e x} /(k T)\left(=\phi_{A} \phi_{B} \chi_{A B}\right.$, see below) denotes the excess free energy contribution due to the enthalpy change of mixing and the entropy change of mixing for the non-random, with respect to random, distribution. $\chi_{S C}$ is known as the structural interaction parameter and can be obtained experimentally either from scattering experiments or by other thermodynamic experiments such as osmometry and sedimentation equilibrium ${ }^{3 a, 12,13)}$. It is determined by the Flory-Huggins interaction parameter, $\chi_{A B}$, its first derivative and the second derivative with respect to composition as discussed by Sanchez ${ }^{3 a)}$ (cf. footnote ${ }^{a}$ ),

$$
\chi_{S C}=\chi_{A B}+\left(\phi_{A}-\phi_{B}\right) \frac{\partial \chi_{A B}}{\partial \phi_{A}}-\frac{\phi_{B} \phi_{A}}{2} \frac{\partial^{2} \chi_{A B}}{\partial \phi_{A}^{2}}
$$

a) It is worth noting that a zero difference in the interaction parameter of a probe with the pure individual polymers does not guarantee a zero difference in the interaction parameter with individual polymers in the mixture, due to the composition dependence of the interaction parameters. 
In IGC ${ }^{26)}$, a mer-mer interaction parameter $\chi_{A B}^{\prime}=\bar{V}_{S} \chi_{A B}=\frac{\bar{V}_{S}}{\bar{V}_{A}} \chi_{A B}^{(F H)}$ is usually used; $\chi_{A B}^{(F H)}\left(=\bar{V}_{A} \chi_{A B}\right)$ is the molecular Flory-Huggins interaction parameter. The equation $\chi_{S C}=\chi_{A B}$ holds if the interaction parameter $\chi_{A B}$ does not depend on the composition.

One may write

$$
\left(\frac{\partial \phi_{A}}{\partial x_{A}}\right)_{T, P}=\frac{\bar{V}_{A} \bar{V}_{B}}{\bar{V}^{2}}
$$

for a binary polymer blend assuming no change of the relative size of the various components with the variation of the composition. Substituting this into Eqs. (3) and (4), one obtains Eqs. (12) and (13) as well as

$$
G_{S A}^{(\infty)}=k T \kappa_{T}-\bar{V}_{S}-\left[\frac{S^{*(A B)}(0)}{\phi_{A}}\right]\left[\left(\frac{1}{\mathrm{kT}} \frac{\partial \mu_{S}^{(M)}}{\partial \phi_{\mathrm{A}}}\right)_{\mathrm{T}, \mathrm{P}}^{\infty}+\frac{1}{\phi_{\mathrm{B}}}\right]
$$

Assuming there is no change of the relative size of macromolecules with the variation of composition, one can obtain the following relationship,

$$
S^{*(A B)}(0)=S^{(A B)}(0)\left(\frac{\bar{V}_{A} \bar{V}_{B}}{\bar{V}}\right)^{2}-\frac{1}{\bar{V}}
$$

By expressing $S^{(A B)}(0)$ with $S^{*(A B)}(0)$ and substituting into Eq. (3), one obtains

$$
\frac{\phi_{A} \phi_{B}}{S^{*(A B)}(0)}\left[G_{S B}^{\infty}(0)-G_{S A}^{\infty}(0)\right]=\lim _{\rho_{S} \rightarrow 0}\left[\frac{\partial \ln \left[\left[^{(w)} \gamma_{S}^{(M)} /^{(w)} \gamma_{S}^{(A)}\right]\right.}{\partial \phi_{A}}\right]
$$

Eq. (13) will be the starting equation for the following discussion. It can be used to discuss the effect of non-random partitioning on IGC data. The integration of Eq. (13) gives the free energy of transfer of the probes between different phases of different compositions, which is also what infinite dilution IGC determines directly from the retention volume of probes ${ }^{\text {b) }}$. It explicitly shows the effect of the non-ran-

b) E.g.,

$$
\left[\ln ^{w} \gamma_{S}^{(M)}\right]^{\infty}=\ln \left[\frac{273.2 R}{P_{S}^{0} V_{g(M)}^{0} M_{S}}\right]-\frac{P_{S}^{0}\left(B_{11}-\bar{V}_{S}^{\infty}\right)}{R T}
$$

or simply

$$
\left[\ln \frac{{ }^{w} \gamma_{S}^{(M)}}{{ }^{w} \gamma_{S}^{(A)}}\right]^{\infty}=-\ln \left[\frac{V_{g(M)}^{0}}{V_{g(A)}^{0}}\right]
$$

assuming no change of $\bar{V}_{S}^{\infty}$ with the blend composition in IGC. $V_{g(M)}^{0}$ is the reduced specific retention volume (see ref. ${ }^{26)}$ ). 
dom partitioning, i.e., $\left[G_{S B}^{\infty}(0)-G_{S A}^{\infty}(0)\right]$. If the Flory-Huggins model is applied to Eq. (13), it will result in the traditional treatment using three interaction parameters of different components ${ }^{4 c)}$.

\section{Local composition and the preferential molecular solvation in a mixture of two polymers}

The preferential solvation can be directly related to the local composition surrounding a solvent molecule, $S$, at its infinite dilution by ${ }^{8,17)}$,

$$
\xi_{S A}=x_{A}\left[1-\frac{x_{B}\left[\left(G_{S B}^{\infty}(0)-G_{S A}^{\infty}(0)\right]\right.}{x_{A} G_{S A}^{\infty}(0)+x_{B} G_{S A}^{\infty}(0)+v_{C V}}\right]
$$

$\xi_{S A}$ denotes the local mole fraction of $\mathrm{A}$ around $\mathrm{S} ; v_{C V}$ is the correlation volume of the polymeric components $\mathrm{A}$ and $\mathrm{B}$ around a probe molecule, $\mathrm{S}$, in the mixture, beyond which the pair correlation functions of $\mathrm{A}$ and $\mathrm{S}$, and $\mathrm{B}$ and $\mathrm{S}$, approach unity. We take $v_{C V}$ to be the molar volume, since we are interested in a mole of the AB mixture. It is worth mentioning that the correlation is short-range, since we are working with the solutions in the very concentrated regime. We may rewrite Eq. (14) as

$$
\frac{\phi_{A}^{*}-\phi_{A}}{\phi_{A} \phi_{B}}=\frac{\left[G_{S B}^{\infty}(0)-G_{S A}^{\infty}(0)\right]}{\left[\phi_{A} G_{S A}^{\infty}(0)\left[\bar{V}_{B} / \bar{V}_{A}\right]+\phi_{B} G_{S B}^{\infty}(0)\right]+\bar{V}_{B}}
$$

assuming that the local volume fraction $\phi_{A}^{*}=\xi_{A} \bar{V}_{A} / \bar{V}$. Substituting Eq. (15) into Eq. (13), one obtains

$-\frac{\phi_{A}^{*}-\phi_{A}}{S^{*(A B)}(0)}\left[\left(\phi_{A} G_{S A}^{\infty}(0)\left[\bar{V}_{B} / \bar{V}_{A}\right]+\phi_{B} G_{S B}^{\infty}(0)\right)+\bar{V}_{B}\right]=\lim _{\rho_{S} \rightarrow 0}\left[\frac{\partial \ln \left[{ }^{(w)} \gamma_{S}^{(M)} /{ }^{(w)} \gamma_{S}^{(A)}\right]}{\partial \phi_{A}}\right]$

The term $\left(\phi_{A} G_{S A}^{\infty}(0)\left[\bar{V}_{B} / \bar{V}_{A}\right]+\phi_{B} G_{S B}^{\infty}(0)\right)\left(\bar{V} / \bar{V}_{B}\right)$ corresponds to the total excess local volume in the bulk of the blend induced by the fluctuation caused by the insertion of solvent molecules (Eq. (14) $)^{8,17)}$. It shows the effect of the difference in molecular sizes of different components on the preferential solvation in the mixture, i.e., the solution asymmetry effect. The total number of the mers does not change much with the variation of the composition (see Eq. (5)) ${ }^{27)}$. At zero preferential solvation, the term $\left(\phi_{A} G_{S A}^{\infty}(0)\left[\bar{V}_{B} / \bar{V}_{A}\right]+\phi_{B} G_{S B}^{\infty}(0)\right)\left(\bar{V} / \bar{V}_{B}\right)$ reduces to $\left(k T \kappa_{T}^{(A B)}-\bar{V}_{S}^{\infty(0)}\right)$, which is small compared to a molar volume of the AB polymer mixture. By choosing systems in which the two components have the same macromolecular lengths, i.e., symmetric mixtures, one obtains $\left(k T \kappa_{T}^{(A B)}-\bar{V}_{S}^{\infty}\right)$ for this term.

\section{Non-random partitioning and inverse gas chromatography experiments}

Measurements are required to determine all of the elements in the chemical potential matrix for a multi-component system (see e.g. Matteoli and Lepori ${ }^{28)}$ ). For poly- 
meric systems, this work will be tedious. The amount of work when using IGC can be significantly reduced by applying limiting conditions. However, rigorous modeling has to be applied for the preferential solvation. It is traditionally accepted that the interaction parameter of the probe with one of the components in the polymeric mixture can be approximated by the interaction parameter of the probe with the component in its pure state. However, the concentration dependence of the interaction parameter may be strong enough so as not to be negligible. For binary systems of a probe, $\mathrm{S}$, with a polymer, $\mathrm{A}$,

$$
G_{S A}^{\infty}(0)=k T \kappa_{T}^{(A)}-\bar{V}_{S}^{\infty}(\mathrm{A})
$$

at infinite dilution of the probe concentration. If we approximate the cluster integrals in a ternary system with the corresponding terms of the individual binary solutions, we obtain the following formula

$$
G_{S B}^{\infty}(0)-G_{S A}^{\infty}(0)=k T\left(\kappa_{T}^{(B)}-\kappa_{T}^{(A)}\right)-\left(\bar{V}_{S}^{\infty(\mathrm{B})}-\bar{V}_{S}^{\infty(\mathrm{A})}\right)
$$

which means a zero preferential solvation if the compressibility term and the difference of the partial molar volume of the probe in different polymers are neglected. Therefore, the traditional approach from classical theory, in fact, is a random partitioning approach applied to non-random partitioning situations. An alternative way of approximating the preferential solvation is to use the cluster integrals of the direct correlation function ${ }^{7}$, which assumes that the composition fluctuation of the blend does not affect non-random partitioning of the probe. However, these approximations are very rough so as to cause discrepancies in the empirical determination.

Experimentally, we can choose a series of probes in infinite dilution IGC (IDIGC) so that $\xi_{A}-x_{A}$ approaches zero and $\frac{\partial \xi_{A}}{\partial x_{A}}=0$. Then Eq. (3) reduces to the following equation $^{\mathrm{c}}$,

$$
\left[S^{*(\mathrm{AB})}(0)\right]^{-1}=\frac{1}{\bar{V}} \lim _{\rho_{\mathrm{S}} \rightarrow 0}\left[\frac{\partial^{2} \ln \left[{ }^{(\mathrm{w})} \gamma_{\mathrm{S}}^{(\mathrm{M})} /^{(\mathrm{w})} \gamma_{\mathrm{s}}^{(\mathrm{A})}\right]}{\partial \phi_{\mathrm{A}}^{2}}\right]^{(0)}
$$

where the script 0 stands for the zero preferential solvation. In IDIGC, the number of solvent probes can be quite easily increased. Different probes may probe the fluctuating bulk differently in terms of spatial distribution. Equations (3), (13), (16) and (19) may be reduced into simple forms according to solvent categories carefully

c) Eq. (3) can be reduced to

$$
\frac{1}{\bar{V}} \lim _{\rho_{s} \rightarrow 0}\left[\frac{\partial^{2} \ln \left[{ }^{(w)} \gamma_{S}^{(M)} /^{(w)} \gamma_{S}^{(A)}\right]}{\partial x_{A}^{2}}\right]
$$

under the random partitioning conditions. This equation can lead to Eq. (19). However, it will lead to an $n$-th order derivative if higher order fluctuations, due to the insertion of the probe molecules, dominate. 
chosen for the experiment in conjunction with appropriate models. This may lessen the experimental effort required to obtain the mer-mer correlation structure factor. To obtain $\mathrm{Eq}$. (3), one must assume that the internal partition (including rotational, vibrational, etc.) does not depend on the composition of the polymeric mixture ${ }^{22)}$. Therefore, small and simple molecules should be used for molecular probe techniques.

\section{A case investigation for IGC applying Eq. (19)}

In order to illustrate the procedure used to obtain polymer-polymer interaction parameters, we applied the formula developed in this paper to a set of experimental data obtained by DiPaola-Baranyi and Degre on polystyrene (A) and poly(butyl methacrylate) (B) blends ${ }^{29)}$.

If a large number of probes and a large number of samples of different composition are used to perform IDIGC, the intersection of the plot of $\frac{\partial^{2} \ln \left[{ }^{(w)} \gamma_{S}^{(M)} \gamma^{(w)} \gamma_{S}^{(A)}\right]}{\partial \phi_{A}^{2}}$ vs. $\frac{\partial \ln \left[(w) \gamma_{S}^{(M)} f^{(w)} \gamma_{S}^{(A)}\right]}{\partial \phi_{A}}$ (which is proportional to the preferential solvation) at zero of $\frac{\partial \ln \left[^{(w)} \gamma_{S}^{(M)} \gamma^{(w)} \gamma_{S}^{(A)}\right]}{\partial \phi_{A}}$ gives $\left[S^{*(A B)}(0) / \bar{V}\right]^{-1}$ (see Eq. (19)), from which the mer-mer correlation structure factor, $S^{*(A B)}(0)$, can be calculated.

However, even though there have been a large number of IGC experiments performed on compatible polymeric blends, these experiments were usually performed for only a few compositions of the blends ${ }^{1,2,30}$. In the case of DiPaola-Baranyi and Degre's experiments, the number of the compositions and the choice of the solvent probes were not large enough for our purpose, which is to obtain the accurate value for the structure factor and the interaction parameter. Therefore, the data presented serve only to illustrate how to use the procedure we suggest in this work. The data regarding the density and average molar mass of the two polymers used here was obtained from literature ${ }^{31)}$. We describe below the determination procedure step by step.

Using the logarithm of the specific retention volume, i.e., $\left[\ln V_{g(M)}^{0}\right]^{\infty}$, obtained from an IGC experiment on different compositions of the polymer blend with a large number of probes, plots of the $-\ln \left[V_{g(M)}^{0} / V_{g(A)}^{0}\right]^{\infty}$ and $-\frac{a^{2} \ln \left[V_{g(M)}^{0} / V_{g(A)}^{0}\right]^{\infty}}{\partial \phi_{A}^{2}}$ vs. $-\frac{\partial \ln \left[V_{g(M)}^{0} / V_{g(A)}^{0}\right]^{\infty}}{\partial \phi_{A}}$ can be established (see Fig. 1). The values of $-\frac{\partial^{2} \ln \left[V_{g(M)}^{0} / V_{g(A)}^{0}\right)^{\infty}}{\partial \phi_{A}^{2}}$ at $-\frac{\partial \ln \left[V_{g(B)}^{0} / V_{g(A)}^{0}\right)^{\infty}}{\partial \phi_{A}}=0$ were used to calculate $\left[S^{*(A B)}(0)\right]^{-1}$ (by Eq. (19), see Fig. 2) ${ }^{\text {d) }}$.

d) The method to determine $\chi_{A B}$ by using the plot of $\frac{\chi_{S(A B)}-\chi_{S(B)}}{\bar{V}_{S}}$ vs. $\frac{\chi_{S(A)}-\chi_{S(B)}}{\bar{V}_{S}}$ at $\frac{\chi_{S(A)}-\chi_{S(B)}}{\bar{V}_{S}}=0$ applying the equation

$$
\left[\frac{\chi_{S(A B)}-\chi_{S(B)}}{\bar{V}_{S}}\right]=\phi_{A}\left[\frac{\chi_{S(A)}-\chi_{S(B)}}{\bar{V}_{S}}\right]-\frac{\chi_{A B} \phi_{A} \phi_{B}}{2}
$$

is the same approach applied for an experiment performed for a single composition of the mixture (see refs. ${ }^{1,2 b, 4 c)}$ ). 
Polymer-polymer interaction parameters by inverse gas chromatography ...

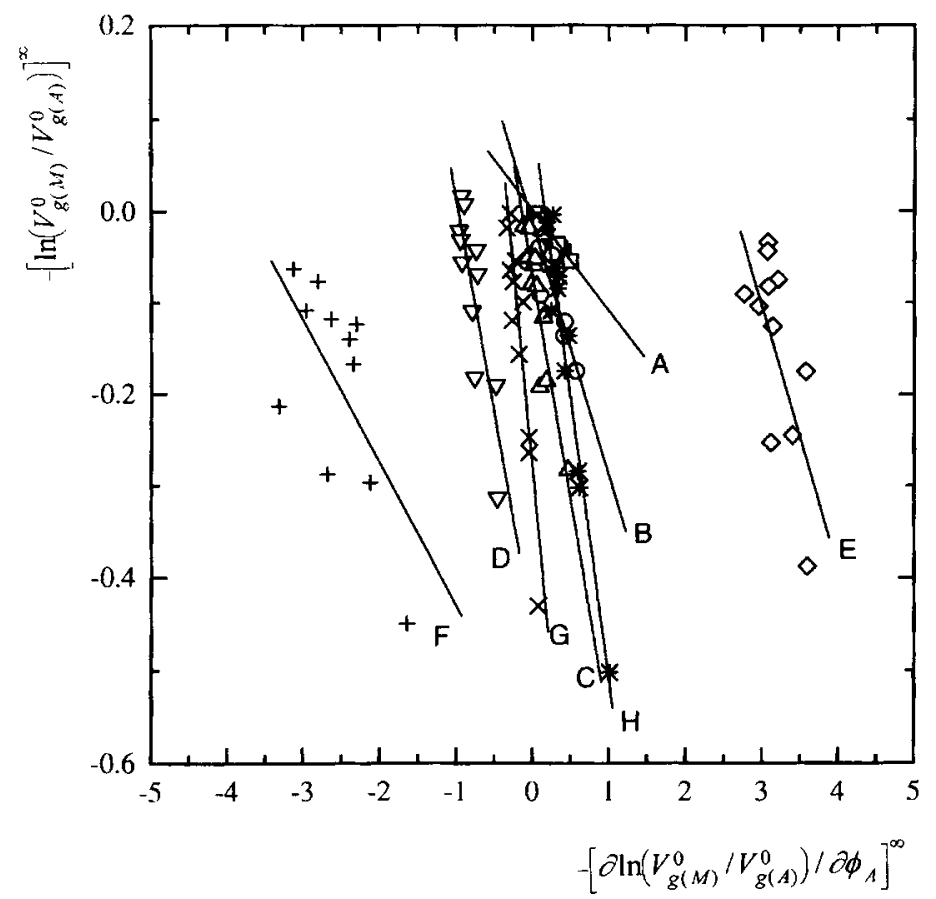

Fig. 1. Plots of the free energy of transfer, $-\left[\ln \left[V_{g(M)}^{0} / V_{g(A)}^{0}\right]^{\infty}\right.$, vs. effective potential gradient, $-\left[\partial \ln \left[V_{g(M)}^{0} / V_{g(A)}^{0}\right) / \partial \phi_{A}\right]^{\infty}$. Data from ref. ${ }^{33)}$. A: $w \%(\mathrm{~A})=90 \%$; B: $w \%(\mathrm{~A})=$ $69 \% ; \mathrm{C}: \mathrm{w} \%(\mathrm{~A})=49 \% ; \mathrm{D}: \mathrm{w} \%(\mathrm{~A})=37.5 \% ; \mathrm{E}: \mathrm{w} \%(\mathrm{~A})=32.5 \% ; \mathrm{F}: \mathrm{w} \%(\mathrm{~A})=28 \% ; \mathrm{G}:$ $\mathrm{w} \%(\mathrm{~A})=21 \% ; \mathrm{H}: \mathrm{w} \%(\mathrm{~A})=7.5 \%$. (w\% (A) means weight percentage of polystyrene)

Fig. 2. The reciprocal of the mer-mer structure factor in $\mathrm{mol} / \mathrm{m}^{3},\left[S^{*(A B)}(0)\right]^{-1}$, vs. the volume fraction of PS (A), $\phi_{\mathrm{A}}$

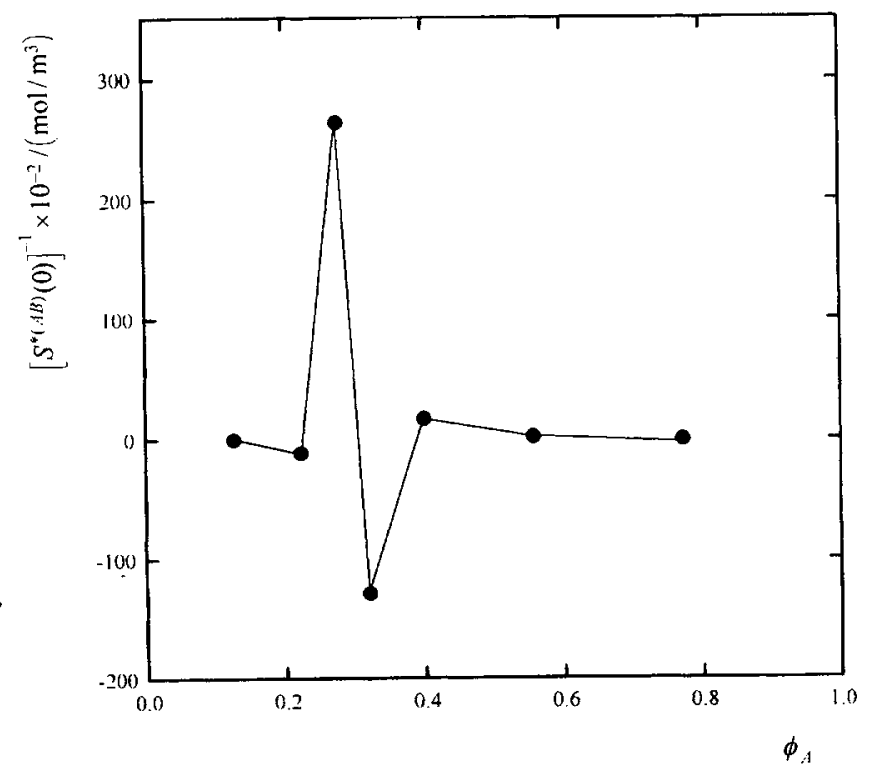


The unit of $\left(\mathrm{mol} / \mathrm{m}^{3}\right)$ is different, by a factor of $10^{-6}$, from the usual units of $\left(\mathrm{mol} / \mathrm{cm}^{3}\right)$ used in the data reporting on neutron scattering experiments ${ }^{13)} \cdot \vec{V}$ was calculated by assuming volume additivity in the mixtures. The structural interaction parameter $\chi_{S C}$ was calculated from the structure factor applying Eq. (9) (see Fig. 3), which, in turn, can be used to calculate the interaction parameter, $\chi_{A B}$, by integrating Eq. (11). To convert this value to the mer-mer interaction parameter, $\chi_{A B}^{\prime}$, used in most IGC papers, a factor of $\bar{V}_{S}$ must be applied.

Fig. 1 shows a linear relationship between $\frac{\partial \ln \left[^{(w)} \gamma_{S}^{(M)} \gamma^{(w)} \gamma_{s}^{(A)}\right]}{\partial \phi_{A}}$ and $\ln \left[{ }^{(w)} \gamma_{S}^{(M)} /^{(w)} \gamma_{s}^{(A)}\right]$, especially for probes close to the random partitioning ${ }^{1,2,4 c, 27)}$. Figs. 1,2 and 3 show an abrupt change of the solution behavior between 0.2 and 0.5 of the volume fraction of polystyrene. The negative value of the structure factor in this concentration regime may be due to a microphase separation.

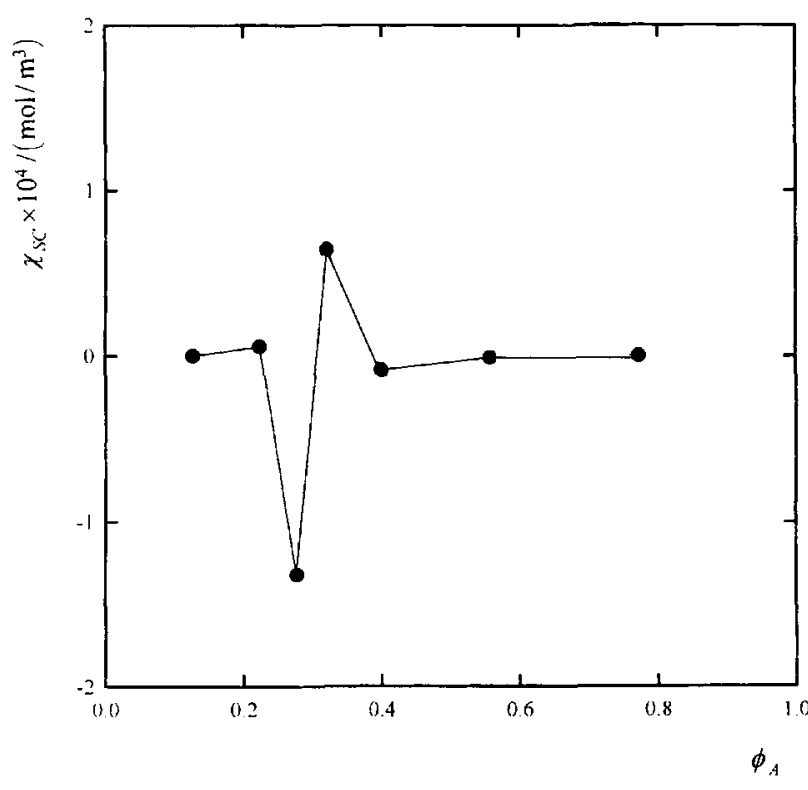

Fig. 3. The structural interaction parameter, $\chi_{S C}$, vs. the volume fraction of $\operatorname{PS}(\mathrm{A}), \phi_{A}$

\section{Conclusion}

Applying the concept of the cluster integrals, the non-random partitioning of solvent probes in a polymeric blend was examined. Eq. (19) gives the direct relationship between the structure factor of a blend, the non-random distribution of probes in the compatible blend, and the experimental quantity that can be obtained from IGC. With the direct link between the structure factor and the interaction parameter from Eqs. (9) and (11) the value of the different interaction parameters can be calculated from experimental data through the structure factor of the blend. This theory offers an alternative way for experimentalists to study the microstructure of polymeric mixtures with IGC. 
We wish to thank Professor J. E. Guillet and Professor S. G. Whittington at the University of Toronto for many enlightening discussions. Funding given by the University of Twente is greatly appreciated. Z. Tan thanks the University of Toronto for the financial support in form of a University of Toronto Open Fellowship and a Connaught Scholarship. We also thank Mrs. Anne Klemperer for her help with the editing of this manuscript.

1) D. R. Lloyd, T. C. Ward, H. P. Schreiber, Eds., "Inverse Gas Chromatography: Characterization of Polymers and Other Materials", ACS Symp. Ser. 391, ACS, Washington, DC 1989

2) H. P. Schreiber, J. C. Berg, Polym. Mater. Sci. Eng. 70, section D, Advances in IGC for Surface Characterization, ACS (1993)

3) a) I. C. Sanchez, Polymer 30, 471 (1989);

b) A. Etxeberria, C. Uriarte, M. J. Fernandez-Berridi, J. J. Iruin, Macromolecules 27, 1245 (1994);

c) A. Etxeberria, M. Iriarte, C. Uriarte, J. J. Iruin, Macromolecules 28, 7188 (1995)

4) a) Z. Y. Al-Saigh, P. Munk, Macromolecules 17, 803 (1984);

b) T. W. Card, Z. Y. Al-Saigh, P. Munk, J. Chromatogr. 18, 1030 (1985);

c) A. M. Farooque, D. D. Deshpande, Polymer 33, 5005 (1992)

5) Z. H. Shi, H. P. Schreiber, Macromolecules 24, 3522 (1991)

6) a) K. S. Schweizer, J. G. Curro, Phys. Rev. Lett. 60, 809 (1988);

b) K. S. Schweizer, J. G. Curro, Adv. Polym. Sci. 116, 319 (1994);

c) U. R. Bidkar, I. C. Sanchez, Macromolecules 28, 3963 (1995)

7) Z. Tan, Ph. D. Thesis, University of Toronto, 1995

8) A. Ben-Naim, Pure Appl. Chem. 62, 25 (1990)

9) K. E. Newman, J. Chem. Soc., Faraday Trans. 1: 84, 1387 (1988)

10) A. Horta, Macromolecules 25, 5651 and 5655 (1992)

11) T. Kato, "Fluctuation Theory of Mixtures", E. Mateoli, G. A. Mansoori, Eds., Taylor \& Francis, New York 1990, p. 227

12) Y. Einaga, Prog. Polym. Sci. 19, 1 (1994)

13) a) C. C. Han, B. J. Bauer, J. C. Clark, Y. Muroga, Y. Matsushita, M. Okada, Q. Trancong, T. Chang, Polymer 29, 2002 (1988);

b) S. Janssen, D. Schwahn, K. Mortensen, T. Springer, Macromolecules 26, 5587 (1993);

c) J. D. Londono, A. H. Harten, G. D. Wignall, K. G. Honnell, E. T. Hsieh, T. W. Johnson, F. S. Bates, Macromolecules 27, 2864 (1994)

14) A. Ben-Naim, "Statistical Thermodynamics for Chemists and Biochemists", Plenum Press, New York 1992

15) a) J. G. Kirkwood, F. P. Buff, J. Chem. Phys. 19, 774 (1951);

b) W. G. McMillan, J. E. Mayer, J. Chem. Phys. 13, 276 (1945)

16) a) J. P. Hansen, I. R. McDonald, "Theory of Simple Liquids", Academic Press, Toronto 1986;

b) D. Gazzillo, Mol. Phys. 84, 303 (1995)

17) E. Z. Hamad, G. A. Mansoori, "Fluctuation Theory of Mixtures", Advances in Thermodynamics vol. 2, E. Matteoli, G. A. Mansoori, Eds., Taylor \& Francis, New York 1990, p. 95

18) H. D. Cochran, L. L. Lee, D. M. Pfund, Fluid Phase Equilib. 34, 219 (1987)

19) A. Ben-Naim, J. Chem. Phys. 63, 2064 (1975)

20) D. M. Pfund, L. L. Lee, H. D. Cochran, Fluid Phase Equilib. 39, 161 (1987)

21) A. Ben-Naim, "Solvation Thermodynamics", Plenum Press, New York 1987 
22) A. Ben-Naim, "Fluctuation Theory of Mixtures", Advances in Thermodynamics vol. 2, E. Matteoli, G. A. Mansoori, Eds., Taylor \& Francis, New York 1990, p. 211

23) a) A. A. Chialvo, "Fluctuation Theory of Mixtures", Advances in Thermodynamics vol. 2, E. Matteoli, G. A. Mansoori, Eds., Taylor \& Francis, New York 1990, p. 131;

b) F. J. Pearson, G. S. Rushbrook, "Fluctuation Theory of Mixtures", Advances in Thermodynamics vol. 2, E. Matteoli, G. A. Mansoori, Eds., Taylor \& Francis, New York 1990, p. 28

24) a) G. Oster, CHem. Rev. 43, 319 (1948);

b) G. A. Miller, J. Phys. Chem. 71, 2305 (1967);

c) M. Kurata, "Thermodynamics of Polymer Solutions", Hardwood Academic Publishers, New York 1982

25) I. C. Sanchez, Macromolecules 24, 908 (1991)

26) D. D. Deshpande, D. Patterson, H. P. Schreiber, S. C. Su, Macromolecules 7, 530 (1974). The term $B=R T \chi_{A B}$ is also often used in the literature (see refs. ${ }^{1)}$ and ${ }^{4 c)}$ )

27) D. Banerjee, A. K. Laha, P. Chatterjee, S. Bagchi, J. Solution. Chem. 24, 301 (1995)

28) E. Matteoli, L. J. Lepori, J. Chem. Soc., Faraday Trans. 91, 431 (1995)

29) G. DiPaola-Baranyi, P. Degre, Macromolecules 14, 1456 (1981)

30) C. G. Smith, P. B. Smith, A. J. Pasztor, L. Jr. McKelvy, D. M. Meunier, S. W. Froelichers, Anal. Chem. 67, 97R (1995)

31) a) H. Höcker, G. J. Blake, P. J. Flory, Trans. Faraday Soc. 67, 2251 (1971);

b) O. Olatisi, R. Simha, Macromolecules 8, 208 (1975) 